\title{
Role of brentuximab vedotin in the treatment of relapsed or refractory Hodgkin lymphoma
}

This article was published in the following Dove Press journal:

Pharmacogenomics and Personalized Medicine

20 February 2014

Number of times this article has been viewed

\section{Tanya Siddiqi \\ Sandra H Thomas \\ Robert Chen}

City of Hope, Department of Hematology and Hematopoietic Cell Transplantation, Duarte, CA, USA
Correspondence: Robert Chen City of Hope Medical Center, I500 E Duarte Rd, Duarte, CA 9I0I0, USA Email rchen@coh.org
Abstract: Brentuximab vedotin (BV) is an antibody-drug conjugate that targets CD30-positive malignancies via an anti-CD30 monoclonal antibody linked to monomethyl auristatin E, a microtubule-disrupting agent, by a protease-cleavable linker. BV has received accelerated approval from the US Food and Drug Administration for the treatment of classical Hodgkin lymphoma that has relapsed either after autologous stem cell transplantation (ASCT) or after two lines of combination chemotherapy in patients ineligible for ASCT, and in systemic anaplastic large cell lymphoma after failure of at least one line of multiagent chemotherapy. Phase I studies in CD30-positive lymphomas have determined the maximum tolerated dose to be $1.8 \mathrm{mg} / \mathrm{kg}$ intravenously every 21 days. In relapsed/refractory Hodgkin lymphoma, a pivotal Phase II study of single-agent BV showed an overall response rate of $75 \%$, with $34 \%$ complete responses and a median remission duration of 20 months for complete responders. BV has a modest toxicity profile, with peripheral neuropathy as one of the most clinically significant side effects, and this is largely reversible. Therefore, BV is the treatment of choice for patients with relapsed/refractory Hodgkin lymphoma after ASCT or two standard regimens. Ongoing trials are evaluating the role of BV as salvage therapy prior to ASCT and for maintenance after ASCT for patients with relapsed/refractory disease.

Keywords: Hodgkin lymphoma, brentuximab vedotin, CD 30, antibody-drug conjugate

\section{Introduction}

Hodgkin lymphoma is thought to arise from germinal center and post-germinal center B-cells, with tumors comprised of a minority of neoplastic (Reed-Sternberg) cells in an inflammatory background of a variable number of lymphocytes, eosinophils, neutrophils, macrophages/histiocytes, plasma cells, fibroblasts, and collagen fibers. There are two distinct types of Hodgkin lymphoma, ie, classical Hodgkin lymphoma and nodular lymphocyte-predominant Hodgkin lymphoma. Classical Hodgkin lymphoma is further divided into four subtypes, ie, nodular sclerosis, mixed cellularity, lymphocyte-rich, and lymphocyte-depleted. Hodgkin lymphoma accounts for about $10 \%$ of all lymphomas and approximately $0.6 \%$ of all cancers; in the USA, there are an estimated 9,000 new cases diagnosed each year. ${ }^{1,2}$

Classical Hodgkin lymphoma is a highly curable cancer with long-term survival exceeding $80 \%{ }^{3-5}$ However, depending on the initial stage and on various prognostic features, up to $30 \%-40 \%$ of patients can relapse after frontline therapy. ${ }^{3-5}$ Standard of care for such patients is salvage chemotherapy followed by autologous stem cell transplantation (ASCT), if eligible for transplant, but this modality confers only about a $50 \%$ remission rate. ${ }^{6}$ For patients relapsing after ASCT, the prognosis is generally poor, with a median overall survival of about $2-2.5$ years. ${ }^{7,8}$ 
Immunophenotypically, virtually all classical Hodgkin lymphoma Reed-Sternberg cells express CD30, a marker that is very useful for the diagnosis of Hodgkin lymphoma. ${ }^{9} \mathrm{CD} 30$ is a transmembrane glycoprotein receptor and is a member of the tumor necrosis factor receptor superfamily. ${ }^{10} \mathrm{CD} 30$ signaling appears to play a role in a variety of biological pathways, including those affecting lymphocyte development and differentiation, as well as those leading to cell proliferation and apoptosis. ${ }^{10,11}$ However, CD30 exhibits very low expression in normal tissues and is generally restricted to activated B-cells, T-cells, and natural killer cells. For these reasons, it is an excellent therapeutic target in classical Hodgkin lymphoma. ${ }^{10}$ Brentuximab vedotin (BV) is an agent that targets CD30 and received accelerated approval from the US Food and Drug Administration in 2011 for the treatment of classical Hodgkin lymphoma that has relapsed either after ASCT, or after two lines of combination chemotherapy in patients ineligible for ASCT, and in systemic anaplastic large cell lymphoma after failure of at least one line of multiagent chemotherapy.

\section{Review of pharmacology, mode of action, and pharmacokinetics}

SGN-30, a chimeric mouse anti-human CD30 antibody, was initially developed to target CD30. In Phase I and II trials, no objective responses were seen in 59 patients with relapsed/refractory classical Hodgkin lymphoma. ${ }^{12,13}$ Seattle Genetics (Bothell, WA, USA) then developed BV (SGN-35, Adcetris $^{\circledR}$ ), an antibody-drug conjugate, to improve responses in patients. BV is comprised of monomethyl auristatin $\mathrm{E}$ (MMAE), a microtubule-disrupting agent, linked to an anti-CD30 monoclonal antibody via a protease-cleavable linker. When this antibody-drug conjugate binds CD30 on the surface of malignant cells, the BV-CD30 complex is internalized into a lysosome where enzymatic linker cleavage releases MMAE. MMAE then binds tubulin and disrupts the microtubules, ultimately leading to $\mathrm{G} 2 / \mathrm{M}$ cell cycle arrest and apoptosis (Figure 1). ${ }^{14}$

In early phase studies, increases in exposure to the antibody-drug conjugate by dose escalation led to approximately proportional exposure to free MMAE. Median time to maximum concentration occurred immediately after infusion for the intact antibody-drug conjugate and about 1-3 days later for MMAE. ${ }^{15,16}$ Steady-state pharmacokinetics for both the antibody-drug conjugate and MMAE occurred by about 21 days and half-life estimates were 4-6 days and 3-4 days, respectively.

\section{Efficacy and toxicity Phase I studies}

Two Phase I trials showed durable objective responses in patients with relapsed/refractory CD30-positive lymphomas (Table 1). ${ }^{15,17}$ Younes et al ${ }^{17}$ reported an open-label, multicenter, dose-escalation study of BV at doses of $0.1-3.6 \mathrm{mg} / \mathrm{kg}$ body weight administered intravenously every 21 days. Fortyfive patients aged 18 years and older were treated in this study, of whom 42 had classical Hodgkin lymphoma. The median number of prior therapies was three, and $73 \%$ of patients had undergone prior ASCT. There was one dose-limiting toxicity at the highest dose level $(3.6 \mathrm{mg} / \mathrm{kg})$ in a patient who developed febrile neutropenia, sepsis, and multiorgan failure, and ultimately died. At the $2.7 \mathrm{mg} / \mathrm{kg}$ dose level, two patients had three dose-limiting toxicities, ie, hyperglycemia, prostatitis, and febrile neutropenia. The maximum tolerated dose was subsequently determined to be $1.8 \mathrm{mg} / \mathrm{kg}$ every 21 days in this study. Thirty-four of 42 evaluable patients showed tumor regression. Objective responses were seen in 17 patients (15 had classical Hodgkin lymphoma), including 11 complete remissions (nine had classical Hodgkin lymphoma). At the maximum tolerated dose, the overall objective response rate was $50 \%$ (six of 12 patients). Median duration of response was at least 9.7 months for all patients and 17.3 months for the 17 responding patients. Toxicities were primarily grade 1 and 2 in severity and the most common adverse events were fatigue (36\%), pyrexia (33\%), diarrhea $(22 \%)$, nausea $(22 \%)$, neutropenia (22\%), and peripheral neuropathy (22\%). The median time to onset of peripheral neuropathy was 9 (range: 3-24) weeks.

Fanale et a $1^{15}$ completed a Phase I dose-escalation study in which BV was administered at doses of $0.4-1.4 \mathrm{mg} / \mathrm{kg}$ to 44 patients with relapsed/refractory CD30-positive lymphomas (38 had Hodgkin lymphoma), on days 1, 8, and 15 of each 28-day cycle for a minimum of two cycles. Patients were aged 12 years and older. The median number of prior therapies was three and $68 \%$ of patients had undergone prior ASCT. One patient experienced a doselimiting toxicity (diarrhea) at the $1 \mathrm{mg} / \mathrm{kg}$ dose level and two patients had dose-limiting toxicities (hyperglycemia and diarrhea) at the $1.4 \mathrm{mg} / \mathrm{kg}$ dose level. Therefore, the maximum tolerated dose was determined to be $1.2 \mathrm{mg} / \mathrm{kg}$ on days 1,8 , and 15 every 28 days in this study. Tumor regression occurred in $85 \%$ of patients and the objective response rate was $59 \%$ (24 patients), with $34 \%$ complete remissions (14 patients) among 41 evaluable patients. The median duration of response was not reached at a median follow-up of 45 weeks on study. The most 
Brentuximab vedotin mechanism of action
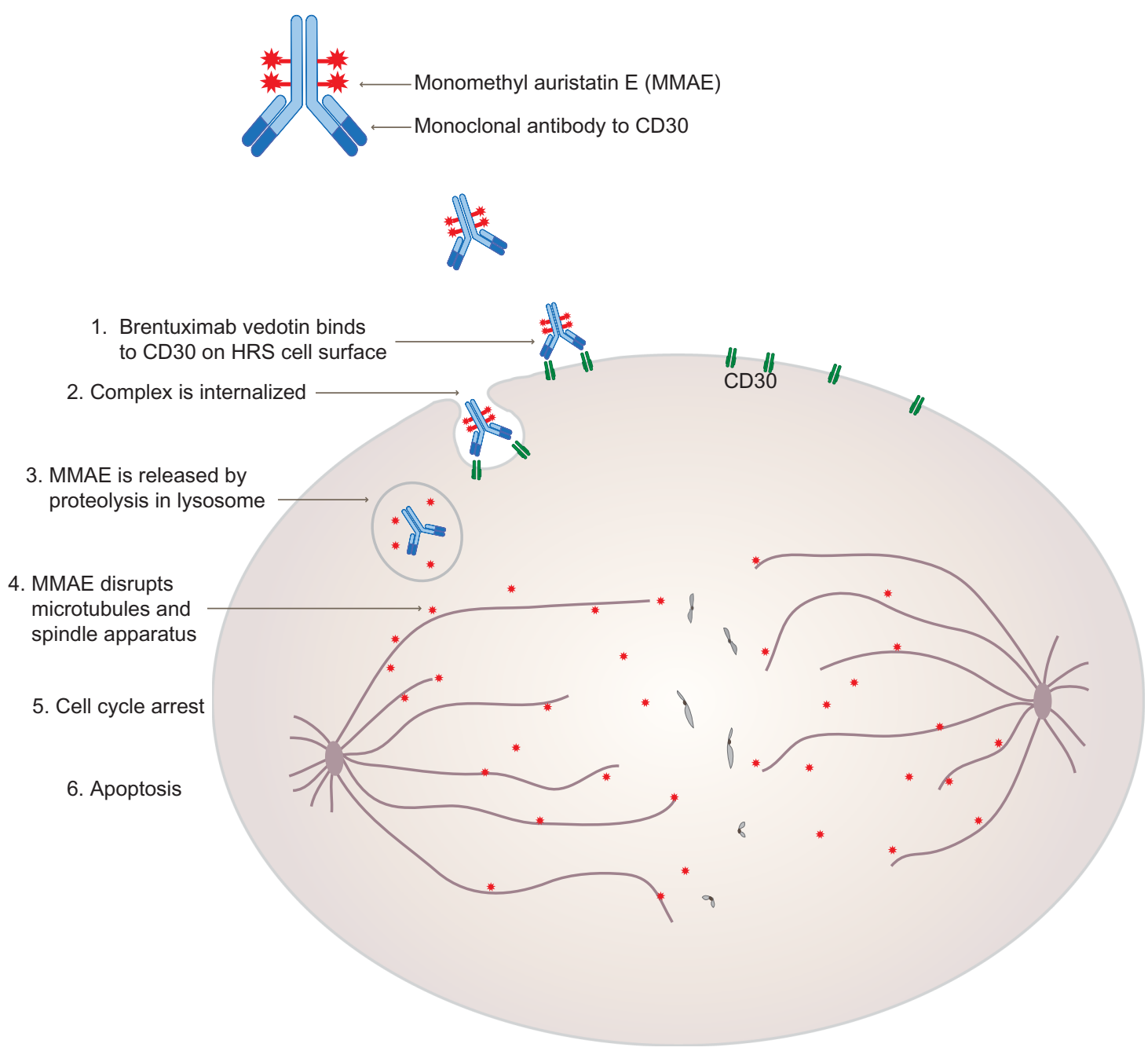

Figure I Mechanism of action of brentuximab vedotin.

Abbreviation: HRS, Hodgkin Reed Sternberg.

common toxicities were peripheral sensory neuropathy $(66 \%)$, fatigue $(52 \%)$, nausea $(50 \%)$, diarrhea $(32 \%)$, arthralgia (27\%), pyrexia (25\%), decreased appetite $(23 \%)$, myalgia (23\%), and upper respiratory tract infection $(23 \%)$. The majority of the adverse events were mild to moderate in severity but $14 \%$ of patients had grade 3 peripheral neuropathy. The median time to onset of any neuropathy event was 6.1 weeks and for grade 3 events was 25.9 weeks. Compared with the every 3 -week treatment schedule, the weekly schedule had similar response rates but higher neurotoxicity rates. Therefore, the weekly schedule was not pursued further in Phase II trials.

Table I Phase I experience with brentuximab vedotin

\begin{tabular}{|c|c|c|}
\hline & Younes et al $^{17}$ & Fanale et al $^{15}$ \\
\hline Patients (n) & 45 & 44 \\
\hline $\mathrm{HL}$ patients & 42 & 38 \\
\hline Median prior therapies (range) & $3(1-7)$ & $3(1-8)$ \\
\hline Patients with prior ASCT (\%) & $33(73 \%)$ & $30(68 \%)$ \\
\hline Dosing schedule & $0.1-3.6 \mathrm{mg} / \mathrm{kg} \mathrm{IV}$ every $2 \mathrm{I}$ days & $0.4-1.4 \mathrm{mg} / \mathrm{kg}$ on days I, 8,15 , every 28 days \\
\hline Tumor regression (\%) & 34 of 42 evaluable patients ( $86 \%)$ & 35 of $4 \mathrm{I}$ evaluable patients ( $85 \%)$ \\
\hline Objective response rate (\%) & $17(40 \%)$ & $24(59 \%)$ \\
\hline Complete remissions (\%) & II (26\%) & 14 (34\%) \\
\hline
\end{tabular}

Abbreviations: ASCT, autologous stem cell transplantation; HL, Hodgkin lymphoma; IV, intravenously. 


\section{Phase II studies}

The pivotal Phase II trial by Younes et al included 102 patients, aged 12 years and older, with centrally reviewed, histologically documented CD30-positive relapsed/refractory Hodgkin lymphoma after ASCT. ${ }^{16}$ This was an international, multicenter, open-label study testing BV at $1.8 \mathrm{mg} / \mathrm{kg}$ intravenously every 21 days for a maximum of 16 cycles. The primary endpoint was objective response rate as determined by an independent review facility. The median number of prior therapies excluding ASCT was 3.5 (range one to 13). Seventy-one percent of patients had primary refractory disease and $42 \%$ had failed to respond to the most recent treatment. All patients had failed ASCT and 71\% had relapsed within 1 year of ASCT. Patients received a median of nine (range one to 16) cycles of therapy. Eighteen patients received all 16 cycles. Tumor reduction was seen in $94 \%$ of patients. The objective response rate was $75 \%$ with $34 \%$ having complete remissions. The median duration of response was 6.7 months for responders and 20.5 months for complete responders. After a median follow-up of 18.5 months, 31 patients were alive and free from disease progression. Median progression-free survival was 5.6 months overall, but in the complete responders it was 21.7 months. The most common adverse events ( $\geq 10 \%$ reports) were peripheral sensory neuropathy (42\%), nausea (35\%), fatigue (34\%), neutropenia (19\%), diarrhea (18\%), pyrexia (14\%), vomiting (13\%), arthralgia (12\%), pruritus $(12 \%)$, myalgia (11\%), peripheral motor neuropathy ( $11 \%)$, and alopecia (10\%). Fifty-five percent of patients experienced grade 3 or higher toxicity: $20 \%$ neutropenia events, $8 \%$ peripheral neuropathy events, and $6 \%$ anemia events. Neuropathy typically developed after prolonged exposure to BV, with a median onset of grade 2 events at 27.3 weeks, which were found to be reversible in most patients. Twenty patients had adverse events that led to treatment discontinuation, including peripheral sensory neuropathy in six patients and peripheral motor neuropathy in three patients. Adverse events in $47 \%$ of patients led to treatment delays, most commonly because of neutropenia (16\%) and peripheral sensory neuropathy (13\%). The dose was reduced to $1.2 \mathrm{mg} / \mathrm{kg}$ every 21 days in eleven patients, ie, ten due to peripheral neuropathy and one due to grade 4 thrombocytopenia.

A small cohort of 25 patients with relapsed Hodgkin lymphoma after allogeneic stem cell transplantation was evaluated in a study by Gopal et al. ${ }^{18}$ These patients were $>100$ days after allogeneic stem cell transplantation, had no active graft versus host disease, and had received a median of nine (range five to 19) prior therapies. Nineteen $(76 \%)$ patients had refractory disease immediately before enrollment on this study. Patients were treated with BV 1.2 $\mathrm{mg} / \mathrm{kg}$ or $1.8 \mathrm{mg} / \mathrm{kg}$ intravenously every 21 days and received a median of eight (range one to 16) cycles. The objective response rate was $50 \%$, with $38 \%$ complete responses among 24 evaluable patients. Duration of response for the complete responders ranged from 5.3 to $>37.1$ weeks. Median progression-free survival was 7.8 months and median overall survival was not reached, although the sample size was too small for the study to make any survival conclusions. The most common adverse events were cough, fatigue, and pyrexia (52\% each), nausea ( $40 \%$ ), peripheral sensory neuropathy (40\%), and dyspnea (40\%). The most frequent adverse events $\geq$ grade 3 in severity were neutropenia (24\%), anemia (20\%), thrombocytopenia (16\%), and hyperglycemia (12\%).

\section{Serious adverse events and their management}

Most adverse events associated with BV are grade 1 or 2 in severity and can be managed with standard supportive care. For more severe adverse events, we recommend the following:

\section{Peripheral neuropathy}

BV-induced peripheral neuropathy is primarily sensory, although motor neuropathy has also been reported. In the pivotal Phase II study for relapsed/refractory Hodgkin lymphoma, ${ }^{16} 42 \%$ of patients experienced peripheral sensory neuropathy of any grade, while $8 \%$ had grade 3 events. Eleven percent of patients in this study experienced peripheral motor neuropathy of any grade, while $1 \%$ had grade 3 events. There were no grade 4 events related to peripheral neuropathy. Twenty-three percent of patients had pre-existing peripheral neuropathy, related in part to prior antimicrotubule chemotherapy exposure, eg, vinca alkaloids. It was found that BV-induced peripheral neuropathy is cumulative and the median time to onset was 12.4 weeks for any grade, 27.3 weeks for grade 2, and 38 weeks for grade 3 peripheral neuropathy. Eighty percent of these patients had resolution of their symptoms, with $50 \%$ having complete resolution at a median of 13.2 weeks after BV was completed/discontinued or the dose reduced. The manufacturer's labeling recommends dose reduction of BV to $1.2 \mathrm{mg} / \mathrm{kg}$ every 21 days for new or worsening grade 2-3 symptoms after the drug has been held to allow the neuropathy to return to grade 1 or lower in severity. BV should be discontinued for grade 4 peripheral neuropathy. Gabapentin and pregabalin have been used in the clinical management of peripheral neuropathy based on anecdotal evidence. 


\section{Neutropenia}

Nineteen percent of patients on the pivotal Phase II trial ${ }^{16}$ developed neutropenia of any grade, while $14 \%$ experienced grade 3 neutropenia and $6 \%$ had grade 4 neutropenia. There were no cases of febrile neutropenia, or any deaths attributable to BV, within 30 days of the last administration of BV. For grade 3-4 neutropenia, prescribing information recommends holding further doses of $\mathrm{BV}$ until resolution to baseline or grade 2 or lower neutrophil count. Growth factor support and frequent laboratory monitoring of the complete blood counts may be considered for subsequent cycles. For recurrent grade 4 neutropenia, dose reduction of BV to $1.2 \mathrm{mg} / \mathrm{kg}$ every 21 days or discontinuation should be considered.

\section{Pancreatitis}

A case series of eight patients, seven with classical Hodgkin lymphoma and one with anaplastic large cell lymphoma, who developed pancreatitis after treatment with single-agent BV was reported at the 2013 American Society of Hematology annual meeting. ${ }^{19}$ Two of these cases were fatal; autopsy was performed on one of these patients and showed acute necrotizing pancreatitis with no evidence of cholelithiasis. The remaining cases were grade 3 or 4 in severity. In seven cases, the dosing of BV was $1.8 \mathrm{mg} / \mathrm{kg}$ every 21 days up to a maximum dose of $180 \mathrm{mg}$. In one case, BV was administered at $1.2 \mathrm{mg} / \mathrm{kg}$ on days $1,8,15$, every 28 days. Median time to onset of pancreatitis from the most recent dose of BV was 12 (range 9-16) days. Two patients were re-treated with BV after resolution of pancreatitis; one had no further evidence of pancreatitis and proceeded to a stem cell transplant, whereas the other patient, having recovered from grade 4 pancreatitis, experienced a second episode (grade 3). All eight patients demonstrated clinical evidence of pancreatitis, as manifested by severe abdominal pain and nausea, and had biochemical and radiologic evidence of pancreatitis with no known underlying history that could have increased their risk of pancreatitis. Abdominal pain can occur in up to $25 \%$ of patients receiving BV. Such patients should be evaluated for pancreatitis, a rare but serious and potentially fatal adverse event related to BV. The authors of this abstract ${ }^{19}$ also recommended considering pretreatment biochemical assessment with serum lipase and/or amylase.

\section{Boxed warning: progressive multifocal leukoencephalopathy}

There have been rare reports of this uncommon complication. ${ }^{20}$ The link to $\mathrm{BV}$ is uncertain given that these patients have been exposed to multiple other agents for their Hodgkin lymphoma as well, most of which can lead to immunosuppression. If multifocal leukoencephalopathy is suspected, BV should be discontinued.

\section{Ongoing clinical trials}

A Phase II trial (NCT01393717) ${ }^{21}$ of BV as salvage therapy at $1.8 \mathrm{mg} / \mathrm{kg}$ intravenously every 21 days for four cycles prior to ASCT is currently in progress. In a similar setting, another Phase II study (NCT01508312) ${ }^{22}$ is evaluating the weekly dosing schedule of BV for two cycles prior to ASCT. A Phase III (NCT01100502) $)^{23}$ trial of BV versus placebo after ASCT has recently completed enrollment, with results pending. In the frontline setting, a Phase III trial (NCT01712490) ${ }^{24}$ is currently underway comparing the standard doxorubicin, bleomycin, vinblastine, dacarbazine (ABVD) regimen with brentuximab vedotin + AVD. Bleomycin has been removed from the BV-containing regimen due to an unacceptably high rate of pulmonary toxicity events (eleven of 25 patients), noted across all dose levels on the brentuximab vedotin $+A B V D$ arm of the Phase I study comparing this regimen with brentuximab vedotin + AVD (zero of 26 patients had pulmonary toxicity). ${ }^{25}$ Another upfront trial (NCT01716806) (26 $^{26}$ is evaluating the role of $\mathrm{BV}$ in older patients with newly diagnosed Hodgkin lymphoma who are not candidates for standard chemotherapy.

$\mathrm{BV}$ can also be used as a bridge to allogeneic stem cell transplantation in responding patients. Chen et al reported a collaborative effort between City of Hope and Fred Hutchinson Cancer Center showing that BV enables successful reduced intensity allogeneic transplantation with a 1 -year progression-free survival of $92 \% .{ }^{27}$ This result was updated at the 12th International Conference on Malignant Lymphoma where a 2-year progression-free survival of $57.5 \%$ and 2-year overall survival of $79 \%$ were reported. ${ }^{28}$ Two ongoing studies are examining the role of BV in the pre-ASCT salvage therapy setting. Chen et al reported their interim results using BV as first-line salvage therapy at the 2012 meeting of the American Society of Hematology, showing an objective response rate of $87.5 \%$ and a complete response rate of $50 \% .{ }^{29}$ Moskowicz et al reported their interim results of sequential BV and chemotherapy (ifosfamide, carboplatin, etoposide) at the 2013 International Conference on Malignant Lymphoma, showing a complete response rate of 33\% with BV alone and a complete response rate of $92 \%$ with the sequential strategy. ${ }^{30}$

\section{Patient-focused issues}

Quality of life and economic evaluations will be incorporated into ongoing and future studies. In general, BV is better 
tolerated than standard combination chemotherapy. Each dose of BV costs $\$ 14,500$, plus potential mark-up by the prescriber. ${ }^{31}$ Confirmation of the benefits of reduced side effects and improved survival rates may offset the disadvantage of the high cost of treatment.

\section{Conclusion}

In the pivotal Phase II trial, ${ }^{16} \mathrm{BV}$ has demonstrated an excellent overall response rate of $75 \%$, with a $34 \%$ complete remission rate among heavily pretreated patients with poor prognosis CD30-positive relapsed/refractory Hodgkin lymphoma. The duration of response for the complete responders is 20.5 months and the median progression-free survival is 21.7 months. The toxicity profile is acceptable, with the most common adverse events being peripheral neuropathy and neutropenia, both largely reversible. Therefore, $\mathrm{BV}$ is the treatment of choice for patients with Hodgkin lymphoma relapsing after ASCT. Several other studies are already underway to expand its use to Hodgkin lymphoma patients at other stages of their disease treatment. A small Phase II study has demonstrated that retreatment with BV at subsequent relapse can result in repeat objective responses, ${ }^{32}$ so retreatment could be considered in patients who do relapse again depending on the depth of their original response to BV. In the extension arm of the same trial, patients were treated for greater than 16 cycles of therapy, for as long as they continued to exhibit responsiveness and tolerable toxicity. At the 2011 American Society of Hematology annual meeting, Forero-Torres et al presented data on 15 patients who have all received $>16$ cycles of $B V .{ }^{33}$ No major changes in the side effect profile was noted and, in fact, there was demonstration of prolonged duration of responses. ${ }^{33}$ The US Food and Drug Administration has changed the indication for duration of drug administration due to positive results from this trial and the package insert for BV no longer contains the 16-cycle limit. Studies are ongoing to examine the role of BV in the pre-ASCT salvage therapy setting as well as in the postASCT maintenance setting for relapsed/refractory Hodgkin lymphoma. This drug, which is in a relatively new category of antibody-drug conjugate, has changed the paradigm of Hodgkin lymphoma treatment in the relapsed/refractory setting. In time, it may alter the landscape for earlier settings of treatment as well.

\section{Acknowledgments}

$\mathrm{RC}$ is supported by the National Cancer Institute of the National Institutes of Health under award number $\mathrm{K} 12 \mathrm{CA} 001727$. The content is solely the responsibility of the authors and does not necessarily represent the official views of the National Institute of Health.

\section{Disclosure}

$\mathrm{RC}$ is a consultant and speaker for Seattle Genetics, and has received research funding from Seattle Genetics. TS is a speaker for Seattle Genetics. The authors have no other conflicts of interest in this work.

\section{References}

1. Jemal A, Siegel R, Ward E, Hao Y, Xu J, Thun MJ. Cancer statistics, 2009. CA Cancer J Clin. 2009;59(4):225-249.

2. Siegel R, Naishadham D, Jemal A. Cancer statistics, 2013. CA Cancer J Clin. 2013;63(1):11-30.

3. Bonadonna G, Bonfante V, Viviani S, Di Russo A, Villani F, Valagussa P. ABVD plus subtotal nodal versus involved-field radiotherapy in early-stage Hodgkin's disease: long-term results. $J$ Clin Oncol. 2004;22(14):2835-2841.

4. Engert A, Diehl V, Franklin J, et al. Escalated-dose BEACOPP in the treatment of patients with advanced-stage Hodgkin's lymphoma: 10 years of follow-up of the GHSG HD9 study. J Clin Oncol. 2009;27(27):4548-4554.

5. Quddus F, Armitage JO. Salvage therapy for Hodgkin's lymphoma. Cancer J. 2009; 15(2):161-163.

6. Majhail NS, Weisdorf DJ, Defor TE, et al. Long-term results of autologous stem cell transplantation for primary refractory or relapsed Hodgkin's lymphoma. Biol Blood Marrow Transplant. 2006;12(10): 1065-1072.

7. Crump M. Management of Hodgkin lymphoma in relapse after autologous stem cell transplant. ASH Education Program Book. 2008;2008(1):326-333.

8. Kewalramani T, Nimer SD, Zelenetz AD, et al. Progressive disease following autologous transplantation in patients with chemosensitive relapsed or primary refractory Hodgkin's disease or aggressive non-Hodgkin's lymphoma. Bone Marrow Transplant. 2003;32(7): 673-679.

9. Swerdlow SH, Campo E, Harris NL, et al. WHO Classification of Tumours of Haematopoietic and Lymphoid Tissues. 4th ed. Geneva, Switzerland: World Health Organization Press; 2008;2.

10. Falini B, Pileri S, Pizzolo G, et al. CD30 (Ki-1) molecule: a new cytokine receptor of the tumor necrosis factor receptor superfamily as a tool for diagnosis and immunotherapy. Blood. 1995;85(1):1-14.

11. Aizawa S, Nakano H, Ishida T, et al. Tumor necrosis factor receptorassociated factor (TRAF) 5 and TRAF2 are involved in CD30-mediated NFkappaB activation. J Biol Chem. 1997;272(4):2042-2045.

12. Bartlett NL, Younes A, Carabasi MH, et al. A phase 1 multidose study of SGN-30 immunotherapy in patients with refractory or recurrent CD30+ hematologic malignancies. Blood. 2008;111(4): $1848-1854$.

13. Forero-Torres A, Leonard JP, Younes A, et al. A Phase II study of SGN-30 (anti-CD30 mAb) in Hodgkin lymphoma or systemic anaplastic large cell lymphoma. Br J Haematol. 2009;146(2):171-179.

14. Sievers EL, Senter PD. Antibody-drug conjugates in cancer therapy. Annu Rev Med. 2013;64:15-29.

15. Fanale MA, Forero-Torres A, Rosenblatt JD, et al. A phase I weekly dosing study of brentuximab vedotin in patients with relapsed/refractory CD30-positive hematologic malignancies. Clin Cancer Res. 2012;18(1): 248-255.

16. Younes A, Gopal AK, Smith SE, et al. Results of a pivotal Phase II study of brentuximab vedotin for patients with relapsed or refractory Hodgkin's lymphoma. J Clin Oncol. 2012;30(18):2183-2189.

17. Younes A, Bartlett NL, Leonard JP, et al. Brentuximab vedotin (SGN-35) for relapsed CD30-positive lymphomas. $N$ Engl J Med. 2010;363(19):1812-1821. 
18. Gopal AK, Ramchandren R, O'Connor OA, et al. Safety and efficacy of brentuximab vedotin for Hodgkin lymphoma recurring after allogeneic stem cell transplantation. Blood. 2012;120(3):560-568.

19. Evens AM, Fenske TS, Hamlin P, et al. Pancreatitis in patients treated with brentuximab vedotin: a previously unrecognized serious adverse event. Blood. 2013;122(21):4380.

20. Wagner-Johnston ND, Bartlett NL, Cashen A, Berger JR. Progressive multifocal leukoencephalopathy in a patient with Hodgkin lymphoma treated with brentuximab vedotin. Leuk Lymphoma. 2012;53(11):2283-2286.

21. City of Hope Medical Center. Brentuximab Vedotin Before Autologous Stem Cell Transplant in Treating Patients With Hodgkin Lymphoma. In: ClinicalTrials.gov [website on the Internet]. Bethesda, MD: US National Library of Medicine; 2011 [updated December 19, 2013]. Available from http://clinicaltrials.gov/show/NCT01393717. NLM identifier: NCT01393717. Accessed January 31, 2014.

22. Memorial Sloan-Kettering Cancer Center. Brentuximab Vedotin (SGN35) in Transplant Eligible Patients With Relapsed or Refractory Hodgkin Lymphoma. In: ClinicalTrials.gov [website on the Internet]. Bethesda, MD: US National Library of Medicine; 2012 [updated October 16, 2013]. Available from http://clinicaltrials.gov/ct2/show/NCT015083 12?term=NCT01508312\&rank=1. NLM identifier: NCT01508312. Accessed January 31, 2014.

23. Seattle Genetics, Inc. A Phase 3 Study of Brentuximab Vedotin (SGN35) in Patients at High Risk of Residual Hodgkin Lymphoma Following Stem Cell Transplant (The AETHERA Trial). In: ClinicalTrials. gov [website on the Internet]. Bethesda, MD: US National Library of Medicine; 2010 [updated January 13, 2014]. Available from http:// clinicaltrials.gov/ct2/show/NCT01100502?term $=$ NCT01100502\&ran $\mathrm{k}=1$. NLM identifier: NCT01100502. Accessed January 31, 2014.

24. Millennium Pharmaceuticals, Inc. Phase 3 Frontline Therapy Trial in Patients With Advanced Classical Hodgkin Lymphoma. In: ClinicalTrials.gov [website on the Internet]. Bethesda, MD: US National Library of Medicine; 2014 [updated January 6, 2014]. Available from http://clinicaltrials.gov/ct2/show/NCT01712490?term=NCT01712490\& rank=1. NLM identifier: NCT01712490. Accessed January 31, 2014.

25. Younes A, Connors JM, Park SI, et al. Brentuximab vedotin combined with ABVD or AVD for patients with newly diagnosed Hodgkin's lymphoma: a phase 1, open-label, dose-escalation study. Lancet Oncol. 2013;14(13):1348-1356.
26. Seattle Genetics, Inc. A Study of Brentuximab Vedotin in Adults Age 60 and Above With Newly Diagnosed Hodgkin Lymphoma (HL). In: ClinicalTrials.gov [website on the Internet]. Bethesda, MD: US National Library of Medicine; 2012 [updated December 20, 2013]. Available from http://clinicaltrials.gov/show/NCT01716806. NLM identifier: NCT01716806. Accessed January 31, 2014.

27. Chen R, Palmer JM, Thomas SH, et al. Brentuximab vedotin enables successful reduced-intensity allogeneic hematopoietic cell transplantation in patients with relapsed or refractory Hodgkin lymphoma. Blood. 2012;119(26):6379-6381.

28. Chen R, Forman SJ, Palmer J, et al. Two-year follow-up of patients with relapsed/refractory Hodgkin treated with brentuximab vedotin prior to reduced intensity allogeneic hematopoietic cell transplantation. Presented at the 12th International Conference on Malignant Lymphoma, Lugano, Switzerland, June 19-22, 2013.

29. Chen RW, Palmer J, Siddiqi T, et al. Brentuximab vedotin as first line salvage therapy in relapsed/refractory HL. ASH Annual Meeting Abstracts. 2012;120(21):3699.

30. Moskowitz AJ, Schoder H, Gerecitano J, et al. PET-adapted sequential therapy with brentuximab vedotin and augmented-ICE induces FDG-PET normalization in $92 \%$ of patients with relapsed and refractory Hodgkin lymphoma. Presented at the 12th International Conference on Malignant Lymphoma, Lugano, Switzerland, June 19-22, 2013.

31. Goyal SD, Bartlett NL. Where does brentuximab vedotin fit into the management of patients with Hodgkin lymphoma? Curr Hematol Malig Rep. 2012;7(3):179-185.

32. Bartlett N, Brice P, Chen RW, et al. Retreatment with brentuximab vedotin in CD30-positive hematologic malignancies: A phase II study. ASCO Meeting Abstracts. 2012;30(Suppl 15):8027.

33. Forero-Torres A, Berryman RB, Advani RH, et al. Prolonged treatment with brentuximab vedotin (SGN-35) in patients with relapsed or refractory hodgkin lymphoma (HL) or systemic anaplastic large cell lymphoma (sALCL). ASH Annual Meeting Abstracts. 2011; 118(21):3711.
Pharmacogenomics and Personalized Medicine

\section{Publish your work in this journal}

Pharmacogenomics and Personalized Medicine is an international, peerreviewed, open access journal characterizing the influence of genotype on pharmacology leading to the development of personalized treatment programs and individualized drug selection for improved safety, efficacy and sustainability. This journal is indexed on the American Chemical

\section{Dovepress}

Society's Chemical Abstracts Service (CAS). The manuscript management system is completely online and includes a very quick and fair peer-review system, which is all easy to use. Visit http://www.dovepress. com/testimonials.php to read real quotes from published authors. 